\title{
Bio-optimized Curcuma longa extract is efficient on knee osteoarthritis pain: a double-blind multicenter randomized placebo controlled three-arm study
}

\author{
Y. Henrotin ${ }^{1,2,3^{*}}$ (D, M. Malaise ${ }^{4}$, R. Wittoek ${ }^{5}$, K. de Vlam $^{6}$, J.-P. Brasseur ${ }^{7}$, F. P. Luyten ${ }^{8}$, Q. Jiangang ${ }^{9}$,
} M. Van den Berghe ${ }^{10}$, R. Uhoda ${ }^{11}$, J. Bentin ${ }^{12}$, T. De Vroey ${ }^{13}$, L. Erpicum ${ }^{14}$, A. F. Donneau ${ }^{15}$ and Y. Dierckxsens ${ }^{16}$

\begin{abstract}
Objectives: Comparison of two doses of bio-optimized Curcuma longa extract (BCL) in the management of symptomatic knee osteoarthritis (OA).

Methods: A prospective, randomized, 3-month, double-blind, multicenter, three-group, placebo-controlled trial assessing Patient Global Assessment of Disease Activity (PGADA) and serum sColl2-1, a biomarker of cartilage degradation, as co-primary endpoints. Pain on visual analog scale (VAS), Knee injury and Osteoarthritis Outcome Score (KOOS), and paracetamol/non-steroidal anti-inflammatory drug (NSAID) consumption were used as secondary endpoints.

Results: One hundred fifty patients with knee OA were followed for 90 days. Low and high doses of BCL showed a greater decrease of PGADA than placebo. Analysis of sColl2-1 showed in the placebo and BCL low-dose groups, but not in the BCL high-dose group, a transient but non-significant increase of sColl2-1 between T0 and T1. Thereafter, in all groups, sColl2-1 decreased between T1 and T3 (all $p<0.01$ ), but no difference between the groups was found. Pain reduction at day 90 in the low- and high-dose $B C L$ groups $(-29.5 \mathrm{~mm}$ and $-36.5 \mathrm{~mm})$ was higher than that in the placebo $(-8 \mathrm{~mm} ; p=0.018)$. The global KOOS significantly decreased overtime, but changes were comparable across treatment arms. The ratio of patients with adverse events (AE) related to the product was similar in the placebo and treatment groups, but the number of AE linked to the product was higher in the high-dose BCL group compared to the placebo $(p=0.012)$.

Conclusions: $\mathrm{BCL}$ appeared safe and well-tolerated with no evidence of severe adverse effects. Efficacy analysis suggested positive trends for measurements of PGADA and serum levels of an OA biomarker and showed a rapid and significant decrease of pain in knee OA (Trial registration: NCT02909621, 21 September 2016 - retrospectively registered).
\end{abstract}

Keywords: Osteoarthritis, Biomarkers, Curcumin

\footnotetext{
*Correspondence: yhenrotin@uliege.be

'Bone and Cartilage Research Unit, Arthropôle Liège, Institute of Pathology,

Level 5, CHU Sart-Tilman, University of Liège, 4000 Liège, Belgium

'Department of Physical Therapy and Rehabilitation, Princess Paola Hospital,

Vivalia, Marche-en-Famenne, Belgium

Full list of author information is available at the end of the article
}

(c) The Author(s). 2019 Open Access This article is distributed under the terms of the Creative Commons Attribution 4.0 International License (http://creativecommons.org/licenses/by/4.0/), which permits unrestricted use, distribution, and reproduction in any medium, provided you give appropriate credit to the original author(s) and the source, provide a link to the Creative Commons license, and indicate if changes were made. The Creative Commons Public Domain Dedication waiver (http://creativecommons.org/publicdomain/zero/1.0/) applies to the data made available in this article, unless otherwise stated. 


\section{Key messages}

- This is the first RCT respecting the clinical guidance investigating the effects of bio-optimized Curcuma longa extract in knee OA patients.

- Bio-optimized Curcuma longa extract is an efficient and safe treatment to relieve pain in knee OA patients.

- Bio-optimized Curcuma significantly decreases paracetamol and non-steroidal anti-inflammatory drug consumption.

\section{Introduction}

Osteoarthritis (OA) affects 240 million people globally, about $10 \%$ of men and $18 \%$ of women [1]. Clinically, OA is characterized by pain, transient morning stiffness, and crepitus with joint motion, all of which deteriorate daily quality of life, leading to sedentary and increased morbidity and mortality $[2,3]$. The prevalence of OA is rising due to the aging population and growing rates of obesity [4]. Up to date, there is no curative treatment for OA despite the availability of a large number of therapeutic options, including non-pharmacological, pharmacological, and surgical therapies. Recommendations for the management of knee OA have been published by numerous scientific and medical societies [5-8]. Briefly, the association of non-pharmacological and pharmacological modalities for optimal management of OA is recommended. The pharmacological treatment of OA is mostly symptomatic to relieve pain and improve function. The first-line pharmacological therapy is the use of paracetamol and non-steroidal anti-inflammatory drugs (NSAIDs). However, these drugs have a low transient analgesic effect on knee OA, and some meta-analysis indicated the occurrence of adverse events mainly in elderly patients with co-morbidities [9-12]. In particular, safety profiles of oral NSAIDs remain a concern, and caution is recommended before selecting an NSAID and dose regimen for a patient [13]. For that reason, it is recommended to use NSAIDs at the lowest efficient dose and intermittently $[8,14]$. The unfavorable safety profiles of commonly prescribed knee OA treatments have led patients, clinicians, and industry to seek safer alternatives including dietary supplements like curcumin $[15,16]$. Curcumin (diferuloylmethane) is the principal curcuminoid extracted from the Curcuma longa (turmeric) root [17]. Curcumin has been identified as an inhibitor of the nuclear factor-kappa $\beta$ (NF-k $\beta)$ pathway and a scavenger of reactive oxygen and nitrogen species $[18,19]$. Two recent meta-analyses evaluating a large number of dietary supplements ranked curcumin as one of the most effective compounds for pain reduction in OA at short term although the quality of evidence was very low [20, 21]. Curcuminoids showed no statistically significant differences in the efficacy outcomes compared to NSAIDs, but patients receiving curcuminoids were significantly less likely to experience gastrointestinal adverse events [21]. A meta-analysis of different turmeric derivatives including curcumin [22] reported the positive influence of biooptimization by adjuvants such as piperine, liposomal delivery systems, phospholipid complexes of curcumin, or use of polysorbate as an emulsifier, which is relevant since curcumin's low bioavailability is regarded as a major challenge for its optimal effectiveness.

Herein, we report results from a study of pharmaceuticalgrade bio-optimized Curcuma longa extract in patients with symptomatic knee OA, which, to our knowledge, is the first ever to have been conducted in full accordance with the International Council for Harmonisation of Technical Requirements for Pharmaceuticals for Human use (ICH E6).

\section{Population and methods \\ Study design and selection of patients}

This study included patients from Belgium enrolled by 11 physicians between September 2014 and June 2017 and was conducted by an independent contract research organization (Artialis SA, Liège, Belgium). The main inclusion criteria were age between 45 and 80 years with primary femorotibial and/or femoropatellar symptomatic knee OA diagnosed according to the clinical and radiological criteria of American College of Rheumatology (ACR) [23]. The more symptomatic knee (with a pain score of at least $40 \mathrm{~mm}$ on a $0-100-\mathrm{mm}$ visual analog scale (VAS) for at least 3 months before enrollment) was defined as the target knee. The main exclusion criteria were dementia or inability to give informed consent, pregnancy or lactation, planned knee replacement surgery, allergy or contraindication to curcumin, recent trauma of the knee responsible of the symptomatic knee, and joint disease other than OA. Use of any intra-articular injection in the target knee in the last 3 months, symptomatic slow-acting drugs in OA (SYSADOA) in the last month, oral corticotherapy $\geq 5 \mathrm{mg}$ /day in the last 3 months, products with curcuminoids extract in the last 3 months, anticoagulant (coumarinic compound), and heparin (both relative contraindication for BCL) was also specifically forbidden by the study protocol. Patients had to be regular users of paracetamol and/or oral NSAIDs to manage OA knee pain and were authorized to continue the intake of these drugs during the study. Ethics Committee approval from all participating centers was obtained, and all patients gave their written informed consent to participate.

\section{Treatment assignment}

Patients were randomly assigned to one of the following three groups with a ratio of 1:1:1: (1) placebo $2 \times 3$ caps/ 
day, (2) bio-optimized Curcuma longa extracts (BCL) low dosage $2 \times 2 \mathrm{caps} /$ day plus placebo $2 \times 1$ cap/day, and (3) BCL high dosage $2 \times 3$ caps/day. Capsules were taken twice daily with water, once at breakfast and once at dinner for 3 months. This pharmaceutical-grade BCL has received approval from Belgian Competent Authorities (PL 31/100; Federal Public Service, Health, Food Chain Safety and Environment) and commercialized under the brand name FLEXOFYTOL (Tilman SA, Baillonville, Belgium). The capsule of $\mathrm{BCL}$ available on the market was encapsulated to allow for a double-blind design. The allocation sequence was created by restricted blocking randomization, with a block size of 6 . To achieve a good balance between the treatments delivered to each center, investigation kits were shipped by blocks, which were multiple of 3 . The investigator was asked to distribute the blinded kit by following the randomization sequence, in order to achieve a good balance between the three treatment groups within this center. Each capsule contained $46.67 \mathrm{mg}$ of turmeric rhizome extract (Curcuma longa L.), Polysorbate 80 [E433] as an emulsifier, and citric acid [E330] as an acidity regulator. Placebo capsules contained sunflower seed oil. For rescue analgesia, patients were allowed to take paracetamol $500 \mathrm{mg}$ tablets (maximum dosage $3 \mathrm{~g} /$ day) or oral NSAIDs if paracetamol was insufficient. An appropriate washout period of $24 \mathrm{~h}$ was required before symptom assessment at in-clinic visits. No other pharmacological or non-pharmacological interventions for OA were allowed. Compliance with the study treatments was established by counting unused study products. Usual treatments taken by the patient in other non-OA-related diseases were allowed; anticoagulants (coumarinic compounds) and heparin were forbidden.

\section{Outcome measures}

There were two co-primary endpoints, and both were assessed as the change from baseline, that is, the difference between the enrollment and study conclusion. One endpoint was the Patient Global Assessment of Disease Activity (PGADA) on a $100-\mathrm{mm}$ visual analog scale (VAS). The other endpoint was the serum levels of Coll2-1 (sColl2-1), a specific amino acid sequence located in the triple helicoidal part of type II collagen and considered as a biomarker of cartilage degradation $[24,25]$. sColl2-1 was measured in diluted serum using an enzymelinked immuno-sensitive assay ELISA method (Artialis SA, Liège, Belgium). The secondary endpoints included the patient's estimate of pain on a 100-mm VAS, the Knee injury and Osteoarthritis Outcome Score (KOOS) index, and its subscale scores using a self-administered questionnaire. Paracetamol/NSAID consumption during the month prior to each visit and patients' satisfaction with the treatment using a 5-point ordinal Likert scale (better, little better, none, little lower, or far lower). All adverse events (AE) and abnormal laboratory test results were recorded.

\section{Statistical analysis}

Statistical analyses have been carried out following the recommendations and guidance on statistical principles for clinical trials (Sakpal, 2010; ICH E9) (Additional file 2). The sample size was considering the results obtained in a previous pilot study [26]. We calculated a sample size of 50 patients per group (150 patients in total) based on a difference in Coll2-1 biomarker level of $44.4 \mathrm{nmol} / \mathrm{l}$ with a standard deviation of 53 and a difference in patient assessment of global disease activity of $21 \mathrm{~mm}$ with a standard deviation of 25, an alpha risk of $2.5 \%$ (to take into account multiplicity due to co-primary endpoints), a power of $85 \%$, and a dropout rate of $15 \%$. Mixed model (repeated measures ANCOVA model) followed by post hoc adjustments test was used to evaluate the efficacy of primary and secondary target variables. $p$ values for treatment effect, time effect, and the effect of their interaction were considered significant at the $2.5 \%$ alpha level for co-primary endpoints $(p<0.025)$ and at the $5 \%$ alpha level for the secondary endpoints $(p<0.05)$.

As no difference between the BCL treated groups was seen at baseline and at 3 months and as curcumin levels in the serum were not significantly different between BCL doses, pooled values of the BCL-treated groups were also considered. PGADA and sColl2-1 from baseline to month 3 were compared between the three treatment groups by means of a linear mixed model. In some situations, a log transformation has been considered for achieving normal distribution of the outcome of interest. Within the longitudinal framework, between-group differences have been investigated at each time point using classical post hoc test. Differences in the distribution of qualitative criteria between the treatment groups have been analyzed using the chi-square $\left(\chi^{2}\right)$ test. Compliance has been evaluated by the pill count method, as well as in percentage of the estimated versus theoretical consumption. Post hoc analysis included the longitudinal analyses of sColl2-1, PGADA, VAS pain, and KOOS using raw data and absolute difference; the longitudinal analyses of Coll2-1, PGADA, and patient' estimated pain using pooled values of the BCL-treated groups; and the longitudinal analyses of PGADA and VAS pain using adjusted value for the intake of rescue treatment. All results were considered to be significant at the $5 \%$ critical level $(p<0.05)$. Statistical analyses were carried out using the Statistical Analysis System (SAS version 9.4, SAS Institute Inc., Cary, NC, USA). The following analysis populations were defined for the study: the safety population (SP) includes all patients treated with at least one dose of the investigational product. The safety analysis was based on the safety population. Intention to treat (ITT) 
population includes every subject who is randomized according to randomized treatment assignment. Full analysis set (FAS) population comprises of subjects who have one efficacy basal measure and at least one corresponding post-baseline efficacy measurement for one of the main efficacy variables, regardless of subsequent withdrawal from treatment or deviation from the protocol. Per-protocol (PP) population included all treated subjects who had not suffered any major protocol deviation.

\section{Results}

One hundred fifty patients were randomized and considered eligible for the ITT analysis and 141 for the FAS analysis. Among the FAS population, 49 received a high dose of BCL, 47 received a low dose of $\mathrm{BCL}$, and 45 received placebo (Fig. 1). The cumulative time distribution of withdrawals was similar in the three groups without significant differences in the reasons for withdrawals. At baseline, participants in each group were well-matched (Table 1). Grades II to IV of KL were equally distributed between the three groups, with $99 \%$ of grade II and III. At inclusion, $92.2 \%$ of patients were taking paracetamol and $71.6 \%$ NSAIDs. All the patients were using paracetamol and/or NSAIDs during the study period. The majority $(82.3 \%)$ of paracetamol and NSAID consumers were taking these drugs between 7 to 14 days a month.

ANCOVA model for repeated measures was performed to analyze the two co-primary endpoints as well as the secondary endpoints on FAS and PP populations. Longitudinal differences such as pain relief and improvement of PGADA were detected for all groups, but no significant difference between the treatment was highlighted. Results for FAS population were shown in Additional file 1: Table S1. Nevertheless, post hoc tests revealed interesting results and were presented here after.

Analysis of PGADA on the ITT population revealed a significant improvement in all treatment arms compared with time (all groups $p<0.0001$ ), but the time evolution curves were found comparable (Fig. 2a). Analysis of absolute difference between baseline and time points on the pooled treated patients in FAS revealed a significantly greater reduction of PGADA in treated patients than in the placebo group at T1 and T3 $(p=0.016$ and $p=0.027$, respectively) (Fig. $2 \mathrm{~b}, \mathrm{c}$ ).

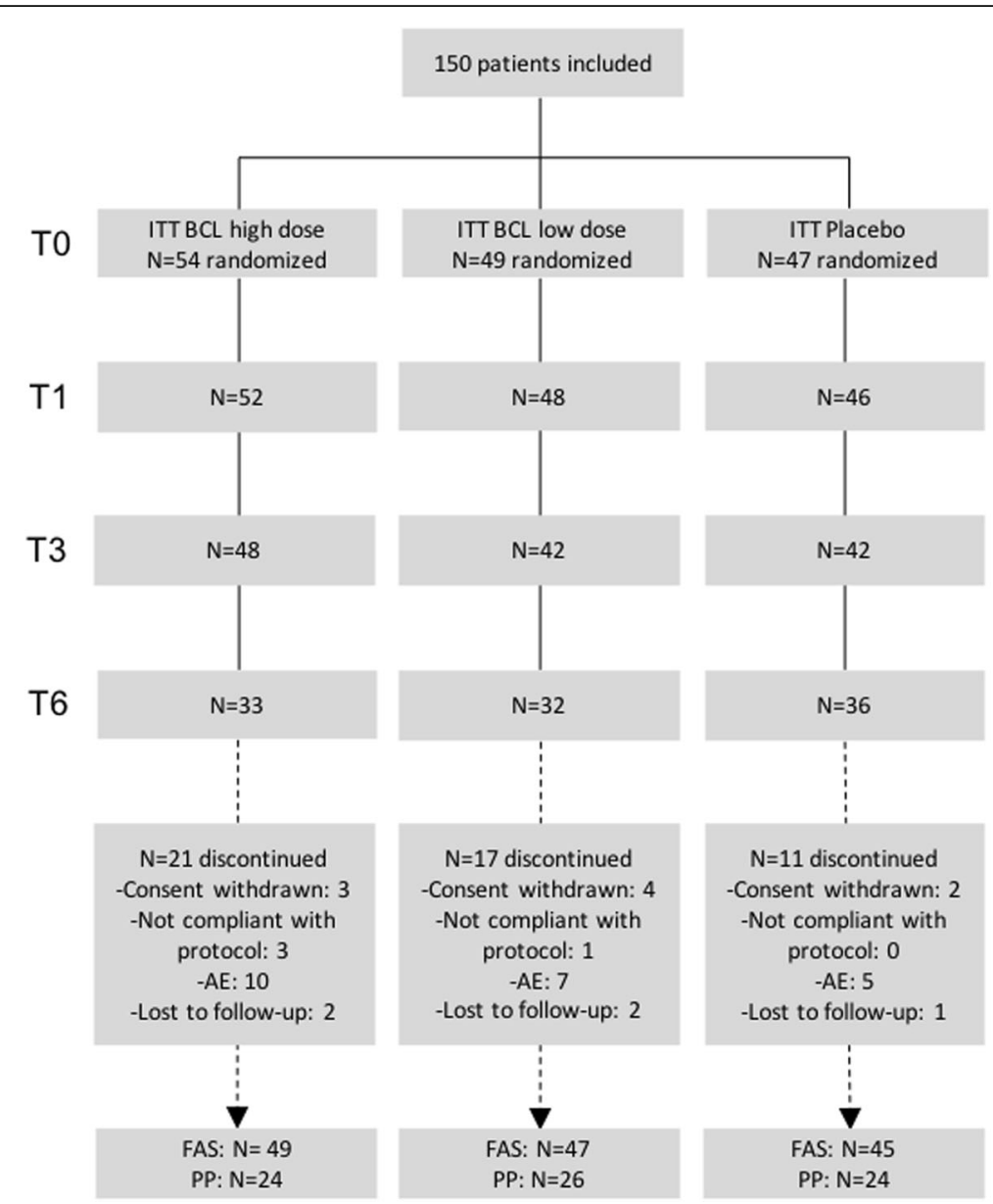

Fig. 1 Disposition of patients. BCL, bio-optimized Curcuma longa; FAS, full analysis set; ITT, intention to treat; PP, per-protocol; $A E$, adverse event 
Table 1 Demographic and baseline characteristics of patients

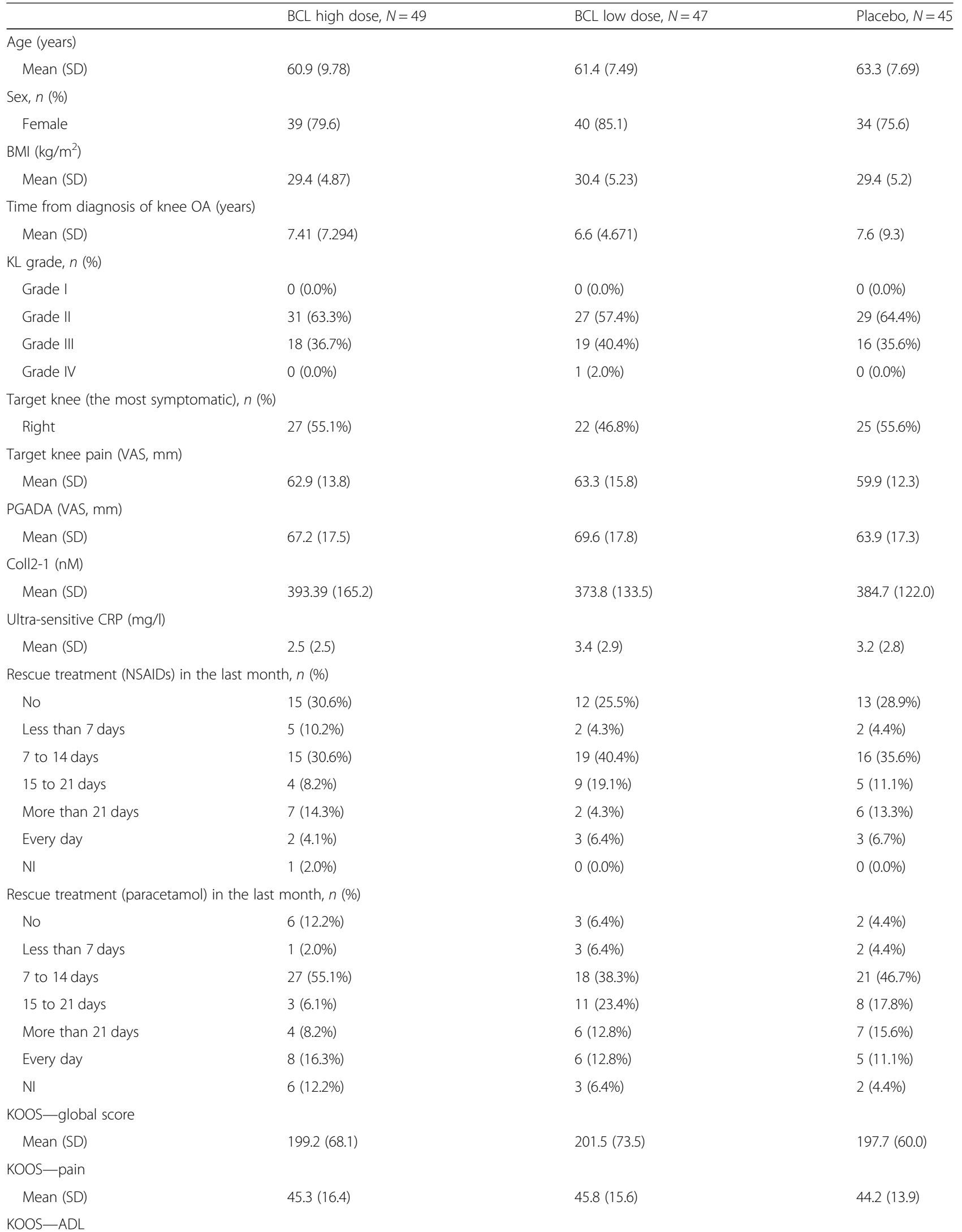


Table 1 Demographic and baseline characteristics of patients (Continued)

\begin{tabular}{|c|c|c|c|}
\hline & $\mathrm{BCL}$ high dose, $N=49$ & BCL low dose, $N=47$ & Placebo, $N=45$ \\
\hline Mean (SD) & $49.8(17.9)$ & $48.9(17.3)$ & $48.9(16.6)$ \\
\hline \multicolumn{4}{|l|}{ KOOS-QoL } \\
\hline Mean (SD) & $32.0(18.7)$ & $31.5(19.2)$ & $33.3(18.1)$ \\
\hline \multicolumn{4}{|c|}{ KOOS—symptoms } \\
\hline Mean (SD) & $52.15(15.9)$ & $52.8(15.6)$ & $54.1(13.5)$ \\
\hline \multicolumn{4}{|c|}{ KOOS_Sport and rec. } \\
\hline Mean (SD) & $19.5(17.1)$ & $19.8(19.8)$ & $17.2(14.5)$ \\
\hline
\end{tabular}

$A D L$ activity of daily life, $B M I$ Body Mass Index, NSAID non-steroidal anti-inflammatory drug, KL Kellgren-Lawrence, NI no information, OA osteoarthritis, PAGDA Patient Global Assessment of Disease Activity, QoL Quality of life, SD Standard Deviation, VAS Visual Analog Scale

Analysis of sColl2-1 on the ITT population showed in the placebo and BCL low-dose groups, but not in the $\mathrm{BCL}$ high-dose group, a transient but non-significant increase of sColl2-1 between T0 and T1. Thereafter, in all groups, sColl2-1 decreased between T1 and T3 (all $p<$ 0.01 ), but no difference between the groups was found (Fig. 3a). Of note, the baseline level of sColl2-1 was similar between the groups. Using pooled values for the treated groups (BCL high plus low doses) on PP population, an absolute difference of sColl2-1 between baseline and T3 revealed a significant difference between the pooled BCL groups and placebo $(P=0.031)$ (Fig. 3b, c).

In ITT, knee pain on VAS significantly decreased with time $(p<0.001)$ in both BCL high and low doses (Fig. 4$)$ while significance was not achieved after 3 months in the placebo group $(p=0.051)$. At T3, a significant difference was observed in ITT between both BCL high dose and low dose and placebo (both $p=0.018$ ), while no significant difference was found between both BCL doses $(p=0.9002)$. The absolute difference between baseline and time points was significantly more important in the $\mathrm{BCL}$ groups than in placebo at $\mathrm{T} 1$ (high dose $-16 \mathrm{~mm}$, low dose $-16.5 \mathrm{~mm}$, placebo -4 $\mathrm{mm} ; p=0.046$ ) and T3 (high dose $-29.5 \mathrm{~mm}$, low dose $-36.5 \mathrm{~mm}$, placebo $-8 \mathrm{~mm} ; p=0.032$ ). The improvement in pain observed in the $\mathrm{BCL}$ low-dose group corresponds to an effect size (ES) of 0.35 for pain at $\mathrm{T} 1$ and 0.43 at $\mathrm{T} 3$.

KOOS global score and subscales were significantly improved over time in all groups $(p<0.001)$, and the evolution was found comparable within the three groups in the FAS population (Table 2). Although KOOS global score and subscale changes to $\mathrm{T} 1$ and $\mathrm{T} 3$ tended to be more important in the BCL groups, no significant difference between the groups was found.

Study of compound usage was $>80 \%$ in all groups and comparable in the placebo and BCL groups, demonstrating excellent compliance. This was confirmed by curcumin measurement in PP. Curcumin serum levels dramatically increased at T1 $(p<0.001)$ and then remained stable until T3 in the low BCL group $(p=$ $0.639)$ while undetectable in the placebo group $(p=$ 0.948)(Fig. 5). At T1, but not at T3, curcumin levels were significantly higher in BCL high dose than in BCL low dose $(p=0.034)$.

Finally, there were significantly more adverse events (AE) related to the product in the BCL high dose than in the $\mathrm{BCL}$ low dose and placebo $(37 \%, 21 \%, 13 \%$,
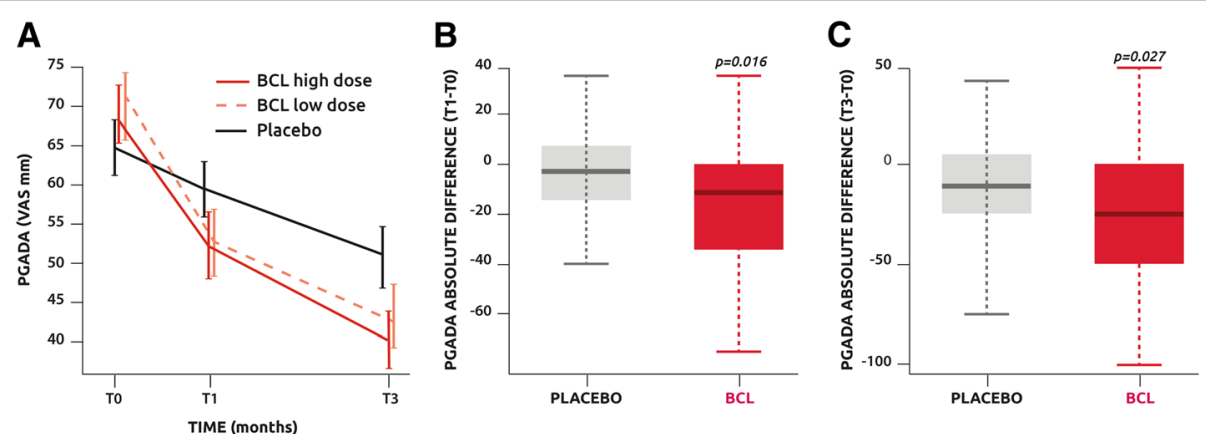

Fig. 2 a Patients Global Assessment of Disease Activity (PGADA) assessed with a VAS evolution with time. $\mathbf{b}$ Comparison of the absolute difference of PGADA between T0 and T1 in the placebo and pooled low- and high-dose BCL groups. c Comparison of the absolute difference of PGDA between T0 and T3 in the placebo and pooled low- and high-dose BCL groups 

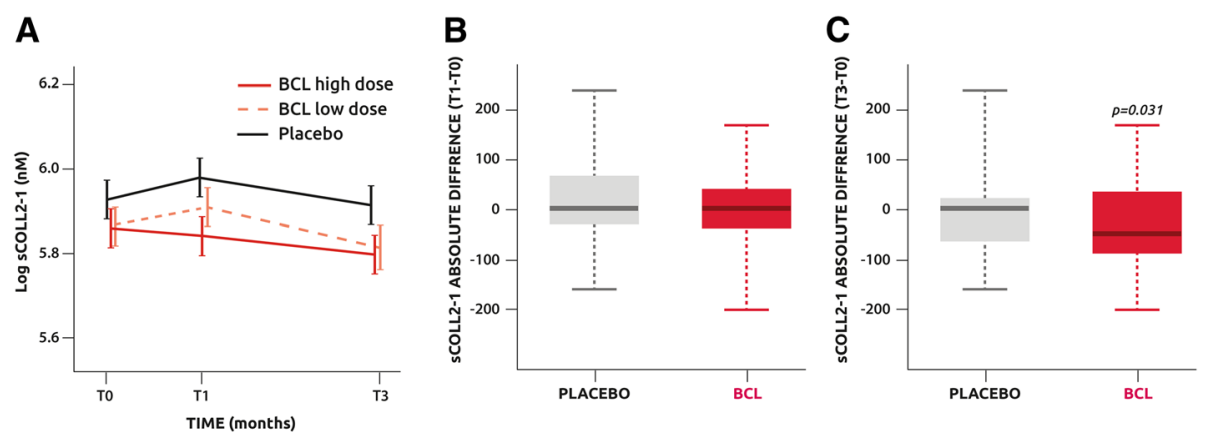

Fig. 3 a Serum levels of Coll2-1 evolution with time. $\mathbf{b}$ Comparison of the absolute difference of sColl2-1 between T0 and T1 in the placebo and pooled low- and high-dose BCL groups. c Comparison of the absolute difference of sCOII2-1 between T0 and T1 in the placebo and pooled lowand high-dose BCL groups

respectively, $p=0.012$ ). No difference between $\mathrm{BCL}$ low dose and placebo was observed. There were no more AE leading to withdrawal in the BCL groups than in placebo. Abdominal discomfort and diarrhea were the most frequently reported $\mathrm{AE}$. Routine laboratory testing did not identify any abnormalities in hepatic or renal dysfunction.

The mean paracetamol or NSAID intake decreased with time in all groups, but no significant difference was observed between the groups at different time points. While global analyses did not reveal any significant difference between the groups at different time points, complementary analyses on FAS population, covering within-group difference, revealed that only subjects of the BCL high-dose group showed a significant decrease in the intake frequency of paracetamol at different time points $(p=0.031$ at T1, $p=0.0016$ at T3) while no variation with time was observed in the low-dose and

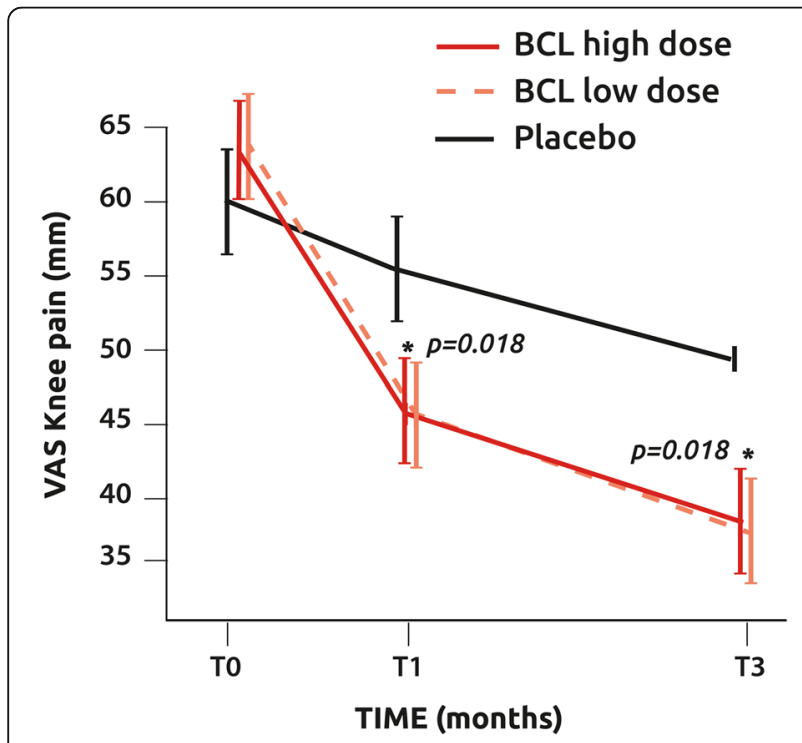

Fig. 4 Knee pain assessment with a VAS evolution with time placebo group. For NSAIDs, a significant decrease of intake frequency was observed only in the low-dose group ( $p=0.038$ at T1, $p=0.029$ at T3).

\section{Discussion}

In this paper, we report the data of a phase II clinical trial investigating the clinical efficacy of two doses of a bio-optimized curcuminoids extract, administered orally during 3 months in patients with symptomatic established knee OA. To our knowledge, this is the first study supporting some therapeutic effect of bio-optimized curcumin in knee OA in a well-designed prospective multicenter trial. Indeed, some recent meta-analyses have pointed out the poor quality of clinical trials investigating food supplements including curcuminoids extracts $[20,21]$. The data indicate that BCL, as an adjuvant treatment to paracetamol and/or NSAIDs, is superior to placebo to decrease pain and PGADA from the first month of treatment. The improvement in pain observed in the BCL low-dose group corresponds to an effect size (ES) of 0.35 for pain at T1 and 0.43 at T3. ES $\leq 0.2$ is usually considered as small while ES between 0.2 and 0.5 is defined as medium. This corroborates the results of previous studies suggesting beneficial effects of curcumin on pain in rheumatoid arthritis [22]. This analgesic effect is all the more remarkable as it is observed in patients who used paracetamol and NSAIDs before and during the study. This means that in our population, BCL has an analgesic effect on top of the expected effect of paracetamol and NSAIDs. Interestingly, NSAID consumption decreased in the low-dose group over time, suggesting that at low dose, BCL decreases pain and NSAID consumption. Considering the adverse effects of NSAIDs, mainly in patients with co-morbidities, BCL could be of value as an alternative to NSAIDs in OA treatment $[27,28]$. Surprisingly, no significant difference was observed with the KOOS. This can be explained by the fact that KOOS was evaluated on a 5-point Likert 
Table 2 KOOS changes between the follow-up visits (T3 and T1) and baseline FAS population

\begin{tabular}{|c|c|c|c|c|}
\hline & & $\begin{array}{l}\mathrm{BCL} \text { high dose, } \\
\text { mean } \pm \mathrm{SD}\end{array}$ & $\begin{array}{l}B C L \text { low dose, } \\
\text { mean } \pm S D\end{array}$ & $\begin{array}{l}\text { Placebo, } \\
\text { mean } \pm \text { SD }\end{array}$ \\
\hline \multirow{7}{*}{$\begin{array}{l}\text { KOOS change at } \mathrm{T} 1 \text { (difference between } \mathrm{T} 1 \\
\text { and baseline) }\end{array}$} & N & 49 & 46 & 45 \\
\hline & Global score & $35.2 \pm 67.5$ & $18.0 \pm 57.6$ & $7.9 \pm 60.2$ \\
\hline & Pain & $7.1 \pm 17.5$ & $4.8 \pm 16.7$ & $3.1 \pm 13.9$ \\
\hline & Symptoms & $6.7 \pm 15.2$ & $2.9 \pm 13.6$ & $1.7 \pm 14.6$ \\
\hline & Function in daily living (ADL) & $5.9 \pm 19.6$ & $4.0 \pm 16.5$ & $1.4 \pm 13.2$ \\
\hline & $\begin{array}{l}\text { Function in sport and recreation } \\
\text { (sport/rec) }\end{array}$ & $10.5 \pm 18.0$ & $4.2 \pm 16.7$ & $2.3 \pm 15.4$ \\
\hline & Knee-related quality of life (QoL) & $5.6 \pm 14.8$ & $3.1 \pm 14.9$ & $-0.2 \pm 16.2$ \\
\hline \multirow{7}{*}{$\begin{array}{l}\text { KOOS change at T3 (difference between T3 } \\
\text { and baseline) }\end{array}$} & N & 47 & 38 & 40 \\
\hline & Global score & $56.3 \pm 82.6$ & 48. \pm 73.1 & $42.1 \pm 66.2$ \\
\hline & Pain & $12.3 \pm 19.4$ & $12.8 \pm 18.4$ & $10.8 \pm 16.5$ \\
\hline & Symptoms & $10.0 \pm 16.6$ & $7.4 \pm 16.0$ & $7.5 \pm 14.7$ \\
\hline & Function in daily living (ADL) & $9.2 \pm 19.5$ & $10.3 \pm 20.9$ & $7.3 \pm 14.6$ \\
\hline & $\begin{array}{l}\text { Function in sport and recreation } \\
\text { (sport/rec) }\end{array}$ & $11.1 \pm 20.5$ & $9.6 \pm 15.3$ & $9.7 \pm 17.8$ \\
\hline & Knee related quality of life (QoL) & $12.4 \pm 20.3$ & $9.2 \pm 19.3$ & $6.6 \pm 16.8$ \\
\hline
\end{tabular}

scale while knee pain was recorded using a visual analog visual scale of $100 \mathrm{~mm}$ which has a better ability to detect clinical change than the Likert scale [29].

One strength of this study was that curcumin levels have been measured in the blood of subjects to evaluate the compliance and the bioavailability of the product. Curcumin levels raised rapidly in the blood of all treated patients while remained undetectable in the placebo group. Another key observation was that the molecule reached a steady state after 1 month. In addition, plateau curcumin levels were comparable in the high- and lowdose BCL groups. This finding provides some indication

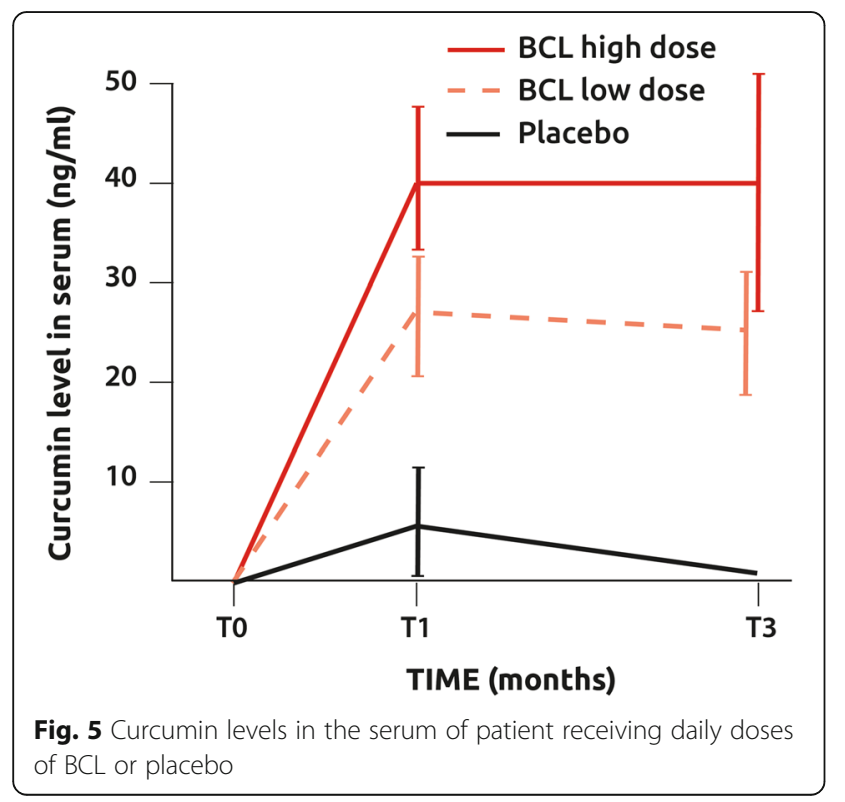

of why both doses showed similar effects on pain. This, combined with the observation that the number of adverse effects related to the product was increased in the high BCL group, justifies the use of low-dose BCL in OA management. This also explains the higher drop-out in the high BCL group. The observed adverse effects were mainly diarrhea. In pharmacological terms, curcumin is a complete choleretic-cholagogue. The cleavage products of curcumin (feluric and hydrofeluric acids) have cholecistokinetic properties because they squeeze the gallbladder, while another principle product, paratolilmethilcarbinol, has a strong choleretic activity. The choleretic effect of curcumin increases bile production by approximately $62 \%$ [30].

Post hoc analyses have also demonstrated that serum Coll2-1, a biomarker of type II collagen degradation, decreased in the BCL groups while slightly increased in the placebo group [24, 25]. Of course, this observation has to be verified in a larger population, and it remains to be demonstrated that this is associated with changes in structural parameters by imaging but suggest that $\mathrm{BCL}$ could downregulate cartilage catabolism and could therefore slow down structural progression.

This study showed some limitations. The main one is the small sample size. It is clear that larger sample sizes are required to confirm the positive trends observed in this study. The second limitation was the particularities of the study population. Indeed, the study population included patients with continuous pain despite the frequent intake of paracetamol and/or NSAIDs thus predominantly non-responders to standard OA pain treatment and thus not very sensitive to anti-inflammatory treatment. 


\section{Conclusions}

In conclusion, this study provided evidence that daily intake of $186.6 \mathrm{mg} /$ day of BCL in patients with symptomatic knee $\mathrm{OA}$ leads to a reduction in pain superior to placebo with a good safety profile and a good compliance, despite the use of paracetamol and/or NSAIDs. Moreover, this trial provides useful information for the design of a larger phase III clinical trial including the sample size estimate, the choice of the dose, and the selection of primary outcomes.

\section{Additional files}

Additional file 1: Table S1. FAS population changes between the follow-up visits (T1, T3, and T6) and baseline and efficacy analysis: results from repeated measures ANCOVA model with post hoc tests if significant. (DOCX $33 \mathrm{~kb}$ )

Additional file 2: Statistical analysis plan. (PDF $1655 \mathrm{~kb}$ )

\section{Abbreviations}

ACR: American College of Rheumatology; AE: Adverse events; BCL: Biooptimized Curcuma longa extracts; ELISA: Enzyme-linked immunosorbent assay; ES: Effect size; FAS: Full analysis set; ICH: International Council for Harmonization; ITT: Intention to treat; KOOS: Knee injury and Osteoarthritis Outcome Score; NF-kß: Nuclear factor-kappa $\beta$; NSAID: Non-steroidal antiinflammatory drug; OA: Osteoarthritis; PGADA: Patient Global Assessment of Disease Activity; SP: Safety population; SYSADOA: Symptomatic slow-acting drugs in OA; VAS: Visual analog scale

\section{Acknowledgements}

Not applicable

\section{Authors' contributions}

$\mathrm{YH}$ conceived the study, participated in its design and coordination, and drafted the manuscript. MM was the principal investigator and recruited the patients. RW, KD, JPB, QJ, MV, RU, JB, TDV, and LE recruited the patients. FL recruited the patients and helped to draft the paper. AFD performed the statistical analysis and helped to draft the manuscript. YD helped to design the study and draft the manuscript. All authors read and approved the final manuscript.

\section{Funding}

All the operational phase of this study (patient recruitment, data collection, and statistical analysis) was funded by Tilman SA

\section{Availability of data and materials}

All the data are available and can be requested at Tilman SA (Baillonville, Belgium, yd@tilman.be).

\section{Ethics approval and consent to participate}

The study protocol was approved by the Central Ethics Committee of the University Hospital of Liège in Belgium (namely Comité d'Ethique HospitaloFacultaire Universitaire de Liège), agreement number: 707. This RCT was also registered on Clinical trial.gov on 13 September 2016 (NCT02909621).

\section{Consent for publication}

Not applicable

\section{Competing interests}

$\mathrm{YH}$ is a consultant for Tilman SA and the founder and chairman of Artialis SA a spin-off company of the University of Liège developing biomarkers. Artialis SA is the manufacturer of coll2-1 immunoassay. YD is an employee of Tilman SA. All other authors declare that they have no competing interests.

\section{Author details}

${ }^{1}$ Bone and Cartilage Research Unit, Arthropôle Liège, Institute of Pathology, Level 5, CHU Sart-Tilman, University of Liège, 4000 Liège, Belgium.

${ }^{2}$ Department of Physical Therapy and Rehabilitation, Princess Paola Hospital, Vivalia, Marche-en-Famenne, Belgium. ${ }^{3}$ Artialis SA, GIGA Tower,

CHU-Sart-Tilman, 4000 Liège, Belgium. ${ }^{4}$ Rheumatology Department, CHU Sart-Tilman, Liège, Belgium. ${ }^{5}$ Rheumatology Department, UZ Gent, Ghent, Belgium. ${ }^{6}$ Rheumatology Department, ZNA Jan Palfijn, Merksem, Belgium. ${ }^{7}$ Rheumatology Department, CHU UCL Namur, Yvoir, Belgium.

${ }^{8}$ Rheumatology Department, University Hospitals Leuven, Leuven, Belgium. ${ }^{9}$ Rheumatology and Physical Medicine Department, Hôpitaux Iris Sud, Bruxelles, Belgium. ${ }^{10}$ Rheumatology Department, Algemeen Stedelijk Ziekenhuis, Aalst, Belgium. ${ }^{11}$ Physical Medicine and Rehabilitation Department, Centre Hospitalier du Bois de l'Abbaye, Seraing, Belgium. ${ }^{12}$ Rheumatology Department, CHU Brugmann, Bruxelles, Belgium. ${ }^{13}$ Physical Medicine, UZA, Antwerpen, Belgium. ${ }^{14}$ Anthisnes, Belgium. ${ }^{15}$ Public health Science Department, University of Liège, Liège, Belgium. ${ }^{16}$ Tilman SA, Baillonville, Belgium.

Received: 15 November 2018 Accepted: 17 July 2019

Published online: 27 July 2019

\section{References}

1. Nelson AE. Osteoarthritis year in review 2017: clinical. Osteoarthritis Cartilage. 2018;26:319-25.

2. Martel-Pelletier J, Barr AJ, Cicuttini FM, et al. Osteoarthritis. Nat Rev Dis Primers. 2016;2:16072.

3. Mendy A, Park J, Vieira ER. Osteoarthritis and risk of mortality in the USA: a population-based cohort study. Int J Epidemiol. 2018;10. https://doi.org/10.1 093/ije/dyy187.

4. Zhang W, Nuki G, Moskowitz RW, et al. OARSI recommendations for the management of hip and knee osteoarthritis: part III: changes in evidence following systematic cumulative update of research published through January 2009. Osteoarthr Cartil. 2010;18:476-99.

5. Jordan KM, Arden NK, Doherty M, et al. Standing Committee for International Clinical Studies Including Therapeutic Trials ESCISIT. EULAR recommendations: an evidence-based approach to the management of knee osteoarthritis: Report of a Task Force of the Standing Committee for International Clinical Studies Including Therapeutic Trials (ESCISIT). Ann Rheum Dis. 2003;62:1145-55.

6. Zhang W, Doherty M, Leeb BF, Alekseeva L, Arden NK, Bijlsma JW, Dincer F, Dziedzic K, Hauselmann HJ, Kaklamanis P, Kloppenburg M, Lohmander LS, Maheu E, Martin-Mola E, Pavelka K. Punzi EULAR evidence-based recommendations for the diagnosis of hand osteoarthritis: report of a task force of ESCISIT. Ann Rheum Dis. 2009;68(1):8-17.

7. McAlindon TE, Bannuru RR, Sullivan MC, et al. OARSI guidelines for the non-surgical management of knee osteoarthritis. Osteoarthr Cartil. 2014; 22:363-88.

8. Doherty M, Hawkey C, Goulder M, Gibb I, Hill N, Aspley S, Reader S. A randomised controlled trial of ibuprofen, paracetamol or a combination tablet of ibuprofen/paracetamol in community-derived people with knee pain. Ann Rheum Dis. 2011;70:1534-41.

9. Machado GC, Maher CG, Ferreira PH, et al. Efficacy and safety of paracetamol for spinal pain and osteoarthritis: systematic review and metaanalysis of randomised placebo-controlled trials. BMJ. 2015;350:h1225. https://doi.org/10.1136/bmj.h1225.

10. Ungprasert $\mathrm{P}$, Cheungpasitporn W, Crowson CS, Matteson EL. Individual non-steroidal anti-inflammatory drugs and risk of acute kidney injury: a systematic review and meta-analysis of observational studies. Eur J Intern Med. 2015;26:285-91.

11. Coxib and traditional NSAID Trialists' (CNT) Collaboration, Bhala N, Emberson J, Merhi A, et al. Vascular and upper gastrointestinal effects of non-steroidal anti-inflammatory drugs: meta-analyses of individual participant data from randomised trials. Lancet. 2013:382:769-79.

12. da Costa B, Keichenbach S, Keller N, et al. Effectiveness of non-steroidal antiinflammatory drugs for the treatment of pain in knee and hip osteoarthritis: a network meta-analysis. Lancet. 2016;387:2093-105.

13. Hochberg MC, Altman RD, April KT, et al. American College of Rheumatology 2012 recommendations for the use of nonpharmacologic and pharmacologic therapies in osteoarthritis of the hand, hip, and knee. Arthritis Care Res. 2012;64:465-74. 
14. Ameye LG, Chee WS. Osteoarthritis and nutrition. From nutraceuticals to functional foods: a systematic review of the scientific evidence. Arthritis Res Ther. 2006;8(4):127-35.

15. Henrotin Y, Lambert C, Couchourel D, Ripoll C, Chiotelli E. Nutraceuticals: do they represent a new era in the management of osteoarthritis? A narrative review from the lessons taken with five products. Osteoarthr Cartil. 2011;19:1-21.

16. Mathy-Hartert M, Jacquemond-Collet I, Priem F, Sanchez C, Lambert C, Henrotin Y. Curcumin inhibits pro-inflammatory mediators and metalloproteinase-3 production by chondrocytes. Inflamm Res. 2009;58:899-908.

17. Wang J, Ma J, Gu JH, Wang FY, Shang XS, Tao HR, Wang X. Regulation of type II collagen, matrix metalloproteinase-13 and cell proliferation by interleukin-1 $\beta$ is mediated by curcumin via inhibition of NF-KB signaling in rat chondrocytes. Mol Med Rep. 2017;16:1837-45.

18. Shen L, Ji HF. The pharmacology of curcumin: is it the degradation products? Trends Mol Med. 2012;18:138-44.

19. Buhrmann C, Mobasheri A, Busch F, Aldinger C, Stahlmann R, Montaseri A, Shakibaei M. Curcumin modulates nuclear factor kappaB (NF-kappaB)mediated inflammation in human tenocytes in vitro: role of the phosphatidylinositol 3-kinase/Akt pathway. J Biol Chem. 2011;286(32): 28556-66.

20. Liu X, Machado GC, Eyles JP, Ravi V, Hunter DJ. Dietary supplements for treating osteoarthritis: a systematic review and meta-analysis. $\mathrm{Br} J$ Sports Med. 2018;52:167-75.

21. Bannuru RR, Osani MC, Al-Eid F, Wang C. Efficacy of curcumin and Boswellia for knee osteoarthritis: systematic review and meta-analysis. Semin Arthritis Rheum. 2018:48(3):416-29.

22. Sahebkar A, Henrotin Y. Analgesic efficacy and safety of curcuminoids in clinical practice: a systematic review and meta-analysis of randomized controlled trials. Pain Med. 2016;17:1192-202.

23. Altman $\mathrm{R}$, Asch $\mathrm{E}$, Bloch $\mathrm{D}$, et al. Development of criteria for the classification and reporting of osteoarthritis. Classification of osteoarthritis of the knee. Diagnostic and Therapeutic Criteria Committee of the American Rheumatism Association. Arthritis Rheum. 1986:29:1039-49.

24. Deberg M, Labasse A, Christgau S, et al. New serum biochemical markers (Coll 2-1 and Coll 2-1 NO2) for studying oxidative-related type II collagen network degradation in patients with osteoarthritis and rheumatoid arthritis. Osteoarthritis Cartilage. 2005;13:258-65.

25. Deberg M, Dubuc JE, Labasse A, et al. One-year follow-up of Coll2-1, Coll2$1 \mathrm{NO} 2$ and myeloperoxydase serum levels in osteoarthritis patients after hip or knee replacement. Ann Rheum Dis. 2008;67:168-74.

26. Henrotin Y, Gharbi M, Dierckxsens Y, et al. Decrease of a specific biomarker of collagen degradation in osteoarthritis, Coll2-1, by treatment with highly bioavailable curcumin during an exploratory clinical trial. BMC Complement Altern Med. 2014;14:159-65.

27. Appelboom T, Maes N, Albert A. A new Curcuma extract (Flexofyto ${ }^{\oplus}$ ) in osteoarthritis: results from a Belgian real-life experience. Open Rheumatol J. 2014;8:77-81.

28. De Breucker $S$, Rouvière $H$, Mélot $C$, Appelboom T. Flexofytol ${ }^{\oplus}$ (a Belgian curcumin extract) for the treatment of aged patients with osteoarthritis and comorbidity. Open J Rheumatol Autoimmune Dis. 2017;7:167-77.

29. du Toit R, Pritchard N, Heffernan S, Simpson T, Fonn D. A comparison of three different scales for rating contact lens handling. Optom Vis Sci. 2002; 79(5):313-20.

30. Wang Y, Wang L, Zhu X, Wang D, Li X. Choleretic activity of turmeric and its active ingredients. J Food Sci. 2016;81:1800-6.

\section{Publisher's Note}

Springer Nature remains neutral with regard to jurisdictional claims in published maps and institutional affiliations.

Ready to submit your research? Choose BMC and benefit from:

- fast, convenient online submission

- thorough peer review by experienced researchers in your field

- rapid publication on acceptance

- support for research data, including large and complex data types

- gold Open Access which fosters wider collaboration and increased citations

- maximum visibility for your research: over $100 \mathrm{M}$ website views per year

At $\mathrm{BMC}$, research is always in progress.

Learn more biomedcentral.com/submissions 